\title{
Joint Position Statement on emergency department overcrowding
}

\author{
Canadian Association of Emergency Physicians and National Emergency Nurses Affiliation
}

\section{Introduction}

Emergency department (ED) overcrowding is a national problem and has become a chronic state in many departments. Overcrowding can be defined as "a situation in which demand for service exceeds the ability to provide care within a reasonable time, causing physicians and nurses to be unable to provide quality care."

While the winter epidemic of respiratory illness results in increased public attention, ED overcrowding reflects complex, systemic problems within the health care system, not just a temporary volume spike during a pandemic influenza season. The Canadian Association of Emergency Physicians (CAEP) and the National Emergency Nurses Affiliation (NENA) would like to outline some of the factors responsible for this problem.

\section{Causes of overcrowding}

\section{Lack of beds for admitted patients}

Increased waiting times for transfer to an inpatient bed has become the most important cause of ED overcrowding. Over the past 5 years, hospital beds in Canada have been reduced by almost $40 \%$ nationally. While some resources have been shifted to home care and long term care, hospitals are having increased difficulty admitting patients from the ED in a timely fashion.

2. Lack of access to primary care, specialist physicians and nurse practitioners

There is a shortage of family physicians, nurse practitioners and many specialists in Canada. In addition, many primary care physicians do not provide after hour access for their patients. Community nursing resources are limited in most regions of Canada, and this situation is expected to worsen over the next 5 years. Patients seek ED care when they do not have a family physician, when they cannot see their physician within an appropriate length of time, or when the waiting time for their specialist, test or procedure becomes too long. As their condition worsens, or if they become frustrated and worried, they seek care in the ED because they know that there is a "specialist" on call and that advanced diagnostic technology is available. Emergency departments have become the "safety net" of the system, but they are sagging under the growing weight.

\section{Shortage of nursing and physician staff}

Manpower studies reveal that there is already a shortage of trained emergency physicians and that our residency programs will not produce enough graduates to fulfill future Canadian needs. Experienced and dedicated nursing staff are the backbone of emergency care, but projections indicate a high likelihood of ongoing nursing shortages. Currently, many Canadian hospitals cannot attract enough nurses to staff their emergency departments. This is partly due to a shortage of qualified nurses and partly because overcrowding has made the ED a frustrating work environment. Increasingly, limited resources prevent emergency staff from providing the care our patients need. Sick patients who need hospital beds languish on emergency stretchers, while suffering patients who need emergency care wait in hallways and waiting rooms. Less often can we provide the care our patients deserve; more often, we find ourselves apologizing for the care they receive.

\section{Increased complexity and acuity of patients presenting to the emergency department}

As the population ages, there is a growing number of patients with chronic conditions such as emphysema, diabetes and cardiovascular disease who require emergency services. These patients often require complex 
assessments utilizing advanced diagnostic technology to determine the need for hospital admission or further therapy. Emergency staff often have the responsibility to develop outpatient follow-up and management plans for patients with acute exacerbation of chronic disease.

\section{Large volumes of patients with non-urgent} problems who could be assessed and treated in another setting

Many people believe that non-urgent patients are a major problem in the ED. In fact, they utilize a small proportion of ED resources and contribute little to ED overcrowding. Concerted efforts to divert these patients to other settings will not solve the problem, will increase costs elsewhere in the health care system and will divert attention from the real problems.

\section{Lack of alternative advanced diagnostic testing and facilities}

The lack of available advanced diagnostic testing in the community causes problems for most emergency departments. In many communities, there are long waits for tests like computed tomography (CT) scans and magnetic resonance imaging (MRI). This causes patients to come to the ED in the hopes of having their investigation done more quickly. In addition, the lack of 24-hour test availability in the ED often forces physicians to hold patients until the next day, when the test is available.

Unavailability of operating room time and delayed access to surgical services increases inpatient and outpatient waiting time, leading to inefficient use of hospital beds and repeated ED visits by patients waiting for treatment.

\section{Effects of overcrowding}

A number of effects have resulted from ED overcrowding.

\section{Inadequate patient care}

As physicians and nurses feel rushed and overextended, the risk of error is increased, and errors could lead to adverse patient outcomes. Although largely anecdotal, recent evidence suggests that overcrowding will lead to medical errors.

\section{Prolonged delays in the treatment of pain and suffering}

With increasing waiting times, patients are kept in stretchers when they should be in beds, and in chairs when they should be in stretchers. Pain relief and improvements in physical, mental and emotional well being are delayed beyond acceptable limits.

\section{Long waiting times and patient dissatisfaction}

With increasing waiting times, a larger number of patients leave without being seen by an appropriate health care provider. Delays in treatment may place patients at risk.

\section{Ambulance diversions}

Ambulance redirect and critical care bypass have become an increasingly common problem in most urban centres in Canada. A number of highly publicized incidents are currently under investigation. Rapid and direct access by the public to emergency care is one of the great strengths of our health care system.

Patients and ambulances should never be denied access to an ED for an urgent or potentially urgent problem.

\section{Decreased nurse/physician satisfaction}

Emergency physicians and nurses feel increasingly responsible to provide care that should be available in the hospital or outpatient setting. This aggravates overcrowding and has a negative effect on ED productivity and morale. As a result, recruitment and retention of experienced, dedicated emergency department staff is a growing problem in Canada.

\section{Negative effect on teaching and research}

In overcrowded departments, ED staff have less time and opportunity to teach and conduct research. Emergency departments have a critical teaching role, which has been compromised by overcrowding. Patient education in the emergency department and the delivery of discharge instructions are also limited by overcrowded conditions.

\section{Solutions to ED overcrowding}

The first critical step is to acknowledge that patients who require hospital admission do not receive adequate care in ED stretchers, and that patients who present to emergency departments with acute medical, surgical or psychiatric conditions deserve appropriate access to ED care. The community and the government must identify ED overcrowding as a high priority health concern and acknowledge that prolonged ED waits for admitted patients are totally unacceptable.

The second step is to recognize that the cause of ED overcrowding generally lies outside the emergency department. 
Efforts to maximize ED efficiency are important, but ED overcrowding is a symptom of system failure, and solutions will require more community care options for the elderly and chronically ill, better access to diagnostic, surgical and acute care services and improved efficiency in the hospital setting.

These solutions will require philosophical and financial support at a government, regional health board and community level.

\section{CAEP and NENA recommend the following strategies}

\section{The primary solution}

Provincial ministries of health should link hospital funding to prompt admission of emergency patients to hospital. Provincial waiting time standards for admitted patients in the ED would need to be developed and enforced. Hospitals may need to open "swing beds" or expand their permanent bed complement to meet waiting time standards. None of the other proposed secondary solutions will have any significant impact unless effective measures are imposed to move patients who require admission, testing or observation out of the emergency department once their initial evaluation and treatment have been completed.

\section{Secondary solutions}

1. Implementation of the Canadian Emergency Department Triage and Acuity Scale (CTAS) in all Canadian emergency departments. Local, provincial, and national reporting structures need to be in place to review data and implement changes to achieve optimum patient care.

2. Implement computer databases so that ED managers can analyze visit volumes, acuity profiles, admission rates, waiting times and lengths of stay. Implement real time Emergency Department Information Systems (EDIS) in all emergency departments so that nurses and physicians can use computerized data to help patients through their ED stay more efficiently. A number of pilot projects are now underway to establish the parameters of such a system. This will enable detailed monitoring of overcrowding and will measure the effect of system changes on patient flow.

3. Link current efforts in primary care reform to ED overcrowding. Emergency department waiting times should be a measure of the success of a primary care initiative. Primary care reform physician registries should have measurements of patient acuity and complexity. The current system entices family physicians to transfer complex patients to the ED.

4. Development of pilot projects in innovative ED care including: rapid diagnostic units, use of a nurse practitioner, bedside registration, point of care testing and linkage to community health centres.

5. Increase access to immediate diagnostic testing (e.g., Doppler ultrasonography, ventilation perfusion (V/Q) scans, CT scans, ultrasonography) to improve patient flow.

6. Expansion of long term care facilities to reduce the number of patients in hospital awaiting placement.

7. Development of innovative home care models to safely discharge patients from the ED and hospital.

8. Expand available training and education programs for emergency physicians and nurses.

9. Create research funding opportunities for emergency physicians and nurses to study the issue of overcrowding in a scientifically valid and timely fashion. The purpose of this research will be to define any regional variation in ED waiting times and to measure the effect of the implementation of information technology in the ED.

10. Develop programs by which the public can be informed about current health care system issues, so they can understand which services can be reasonably expected from an ED.

Correspondence to: Canadian Association of Emergency Physicians, Head Office, 1785 Alta Vista Dr., Ste. 104, Ottawa ON K1G 3Y6; 613 523-3343, fax 613 523-0190, admin@ caep.ca 